\title{
A deep learning-based worker assistance system for error prevention: Case study in a real-world manual assembly
}

\author{
Riedel, A. ${ }^{\mathrm{a},{ }^{*},}$, Gerlach, J. ${ }^{\mathrm{a}}$, Dietsch, M. ${ }^{\mathrm{a}}$, Herbst, S. ${ }^{\mathrm{a}}$, Engelmann, F. ${ }^{\mathrm{a}}$, Brehm, N. ${ }^{\mathrm{a}}$, Pfeifroth, T. ${ }^{\mathrm{a}}$ \\ aDepartment of Industrial Engineering, Ernst-Abbe University of Applied Sciences, Jena, Germany
}

\begin{abstract}
A B S T R A C T
Modern assembly systems adapt to the requirements of customised and short-lived products. As assembly tasks become increasingly complex and change rapidly, the cognitive load on employees increases. This leads to the use of assistance systems for manual assembly to detect and avoid human errors and thus ensure consistent product quality. Most of these systems promise to improve the production environment but have hardly been studied quantitatively so far. Recent advances in deep learning-based computer vision have also not yet been fully exploited. This study aims to provide architectural, and implementational details of a state-of-the-art assembly assistance system based on an object detection model. The proposed architecture is intended to be representative of modern assistance systems. The error prevention potential is determined in a case study in which test subjects manually assemble a complex explosion-proof tubular lamp. The results show $51 \%$ fewer assembly errors compared to a control group without assistance. Three of the four considered types of error classes have been reduced by at least $42 \%$. In particular, errors by omission are most likely to be prevented by the system. The reduction in the error rate is observed over the entire period of 30 consecutive product assemblies, comparing assisted and unassisted assembly. Furthermore, the recorded assembly data are found to be valuable regarding traceability and production improvement processes.
\end{abstract}

\section{ARTICLE INFO}

Keywords:

Deep learning;

Machine learning;

Industry 4.0;

Smart manufacturing;

Manual assembly;

Assistance system;

Error prevention;

Object detection

*Corresponding author:

alexander.riedel@eah-jena.de

(Riedel, A.)

Article history:

Received 14 October 2021

Revised 3 December 2021

Accepted 4 December 2021

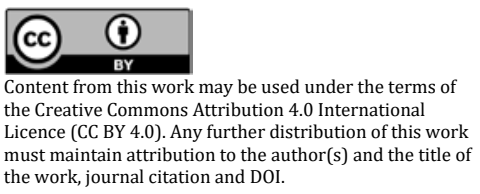

\section{References}

[1] Koren, Y. (2010). The global manufacturing revolution: Product-process-business integration and reconfigurable systems, John Wiley \& Sons, New Jersey, USA, doi: 10.1002/9780470618813.

[2] Zhou, F., Ji, Y., Jiao, R.J. (2013). Affective and cognitive design for mass personalization: Status and prospect, Journal of Intelligent Manufacturing, Vol. 24, No. 5, 1047-1069, doi: 10.1007/s10845-012-0673-2.

[3] Metzmacher, A.I., Hellebrandt, T., Ruessmann, M., Heine, I, Schmitt, R.H. (2019). Aligning the social perspective with the technical vision of the smart factory, In: Schmitt, R., Schuh, G. (eds.), Advances in Production Research, WGP 2018, Springer, Cham, Switzerland, 715-729, doi: 10.1007/978-3-030-03451-1 69.

[4] Merkel, L., Atug, J., Berger, C., Braunreuther, S., Reinhart, G. (2018). Mass customization and paperless assembly in the learning factory for cyber-physical-production systems: Learning module 'from paperbased to paperless assembly', In: Proceedings of 2018 IEEE 18 th International Conference on Advanced Learning Technologies (ICALT), Mumbai, India, 270-271, doi: 10.1109/ICALT.2018.00130.

[5] Villani, V., Sabattini, L., Czerniaki, J.N., Mertens, A., Vogel-Heuser, B., Fantuzzi, C. (2017). Towards modern inclusive factories: A methodology for the development of smart adaptive human-machine interfaces, In: Proceedings of 2017 22 nd IEEE International Conference on Emerging Technologies and Factory Automation (ETFA), 1-7, doi: 10.1109/ETFA.2017.8247634.

[6] Quint, F., Loch, F., Orfgen, M., Zuehlke, D. (2016). A system architecture for assistance in manual tasks, In: Novais, P., Konomi, S. (eds.), Ambient intelligence and smart environments, Volume 21: Intelligent Environments, IOS Press BV, Amsterdam, The Netherlands, 43-52, doi: 10.3233/978-1-61499-690-3-43. 
[7] Fast-Berglund, Å., Fässberg, T., Hellman, F., Davidsson, A., Stahre, J. (2013). Relations between complexity, quality and cognitive automation in mixed-model assembly, Journal of Manufacturing Systems, Vol. 32, No. 3, 449-455, doi: 10.1016/j.jmsy.2013.04.011.

[8] Hinrichsen, S., Bendzioch, S. (2019). How digital assistance systems improve work productivity in assembly, In: Nunes, I.L. (ed.), Advances in Human Factors and Systems Interaction, Springer International Publishing, 332-342, doi: $10.1007 / 978-3-319-94334-333$.

[9] Turk, M., Pipan, M., Šimic, M., Herakovič, N. (2020). Simulation-based time evaluation of basic manual assembly tasks, Advances in Production Engineering \& Management, Vol. 15, No. 3, 331-344, doi: 10.14743/apem2020.3. $\underline{369}$.

[10] Zamora-Hernández, M.-A., Castro-Vargas, J.A., Azorin-Lopez, J., Garcia-Rodriguez, J. (2021). Deep learning-based visual control assistant for assembly in Industry 4.0, Computers in Industry, Vol. 131, Article No. 103485, doi: 10.1016/i.compind.2021.103485.

[11] Min, Y., Zhang, Y., Chai, X., Chen, X. (2020). An efficient pointLSTM for point clouds based gesture recognition, In: Proceedings of 2020 IEEE/CVF Conference on Computer Vision and Pattern Recognition (CVPR), Seattle, USA, 5760-5769, doi: 10.1109/CVPR42600.2020.00580.

[12] Börold, A., Teucke, M., Rust, A., Freitag, M. (2020). Deep learning-based object recognition for counting car components to support handling and packing processes in automotive supply chains, IFAC-PapersOnLine, Vol. 53, No. 2, 10645-10650, doi: 10.1016/j.ifacol.2020.12.2828.

[13] Ozdemir, R., Koc, M. (2019). A quality control application on a smart factory prototype using deep learning methods, In: Proceedings of 2019 IEEE 14th International Conference on Computer Sciences and Information Technologies (CSIT), Lviv, Ukraine, 46-49, doi: 10.1109/STC-CSIT.2019.8929734.

[14] Pierleoni, P., Belli, A., Palma, L., Palmucci, M., Sabbatini, L. (2020). A machine vision system for manual assembly line monitoring, In: Proceedings of 2020 International Conference on Intelligent Engineering and Management (ICIEM), London, United Kingdom, 33-38, doi: 10.1109/ICIEM48762.2020.9160011.

[15] Tavakoli, H., Walunj, S., Pahlevannejad, P., Plociennik, C., Ruskowski, M. (2021). Small object detection for near real-time egocentric perception in a manual assembly scenario, Computer Science, Computer Vision and Pattern Recognition, Cornell University, from https://arxiv.org/abs/2106.06403, accessed October 17, 2021.

[16] Eversberg, L., Grosenick, P., Meusel, M., Lambrecht, J. (2021). An industrial assistance system with manual assembly step recognition in virtual reality, In: Proceedings of 2021 International Conference on Applied Artificial Intelligence (ICAPAI), Halden, Norway, 1-6, doi: 10.1109/ICAPAI49758.2021.9462061.

[17] Egeler, R., Wimpff, D.-P., Jauch, C., Wiedenroth, S.J., Wolf, A., Ruck, M., Wohlfeld, D. (2017). MonSiKo - Adaptives Montageassistenz- und Interaktionssystem mittels 3D-Szenenanalyse und intuitiver Mensch-Technik Kommunikation, Schlussbericht, Stuttgart, Germany, from http://publica.fraunhofer.de/dokumente/N-590407. html, accessed October 14, 2021.

[18] Oestreich, H., Töniges, T., Wojtynek, M., Wrede, S. (2019). Interactive learning of assembly processes using digital assistance, Procedia Manufacturing, Vol. 31, 14-19, doi: 10.1016/i.promfg.2019.03.003.

[19] Kaczmarek, S., Hogreve, S., Tracht, K. (2015). Progress monitoring and gesture control in manual assembly systems using 3D-image sensors, Procedia CIRP, Vol. 37, 1-6, doi: 10.1016/i.procir.2015.08.006.

[20] Faccio, M., Ferrari, E., Galizia, F.G., Gamberi, M., Pilati, F. (2019). Real-time assistance to manual assembly through depth camera and visual feedback, Procedia CIRP, Vol. 81, 1254-1259, doi: 10.1016/j.procir.2019. $\underline{03.303 .}$.

[21] Pilati, F., Faccio, M., Gamberi, M., Regattieri, A. (2020). Learning manual assembly through real-time motion capture for operator training with augmented reality, Procedia Manufacturing, Vol. 45, 189-195, doi: 10.1016/ j.promfg.2020.04.093.

[22] Rocha, C.A.P., Rauch, E., Vaimel, T., Garcia, M.A.R., Vidoni, R. (2021). Implementation of a vision-based worker assistance system in assembly: A case study, Procedia CIRP, Vol. 96, 295-300, doi: 10.1016/i.procir.2021.01.090.

[23] Bochkovskiy, A., Wang, C.-Y., Liao, H.-Y.M. (2020). YOLOv4: Optimal speed and accuracy of object detection, Computer Science, Computer Vision and Pattern Recognition, Cornell University, from http://arxiv.org/abs/2004.10934, accessed October 14, 2021.

[24] NVIDIA deep learning TensorRT documentation, from https://docs.nvidia.com/deeplearning/tensorrt/developerguide/index.html, accessed October 14, 2021.

[25] Redmon, J., Darknet: Open Source Neural Networks in C, from http://pjreddie.com/darknet// accessed October $14,2021$.

[26] Stockinger, C., Steinebach, T., Petrat, D., Bruns, R., Zöller, I. (2020). The effect of pick-by-light-systems on situation awareness in order picking activities, Procedia Manufacturing, Vol. 45, 96-101, doi: 10.1016/i.promfg. 2020.04.078.

[27] Annett, J. (2003). Hierarchical task analysis, In: Hollnagel, E. (ed.), Handbook of cognitive task design, Vol. 2, Lawrence Erlbaum Associates, Mahwah, New Jersey, USA, 17-35.

[28] Kirwan, B. (1994). A guide to practical human reliability assessment, First edition, CRC Press, London, United Kingdom.

[29] VDI. VDI 4006 Blatt 2:2017-11, Human reliability - Methods for quantitative assessment of human reliability. (2017), from https://www.vdi.de/, accessed October 14, 2021.

[30] Jiang, C., Xi, J.T. (2019). Dynamic scheduling in the engineer-to-order (ETO) assembly process by the combined immune algorithm and simulated annealing method, Advances in Production Engineering \& Management, Vol. 14, No. 3, 271-283, doi: 10.14743/apem2019.3.327. 


\section{APEM}

Advances in Production Engineering \& Management Letnik 16 | Številka 4 | December 2021 | Strani 393-404 https://doi.org/10.14743/apem2021.4.408
ISSN 1854-6250

Spletna stran: apem-journal.org Izvirni znanstveni članek

\title{
Sistem za pomoč delavcem za preprečevanje napak, ki temelji na globokem učenju: Študija primera ročne montaže $v$ realnem industrijskem okolju
}

\author{
Riedel, A. ${ }^{\text {a }}{ }^{,}$, Gerlach, J. ${ }^{a}$, Dietsch, M. ${ }^{a}$, Herbst, S. ${ }^{a}$, Engelmann, F. ${ }^{a}$, Brehm, N. ${ }^{a}$, Pfeifroth, T. ${ }^{a}$ \\ aDepartment of Industrial Engineering, Ernst-Abbe University of Applied Sciences, Jena, Germany
}

\section{POVZETEK}

Sodobni montažni sistemi se prilagajajo zahtevam prilagojenih izdelkov s kratkimi življenjskimi dobami. Ker postajajo montažne naloge vse bolj zapletene in se hitro spreminjajo, se povečuje kognitivna obremenitev zaposlenih. To vodi do uporabe podpornih sistemov za odkrivanje in izogibanje človeškim napakam pri ročni montaži ter pripomore k zagotavljanju dosledne kakovosti izdelkov. Večina teh sistemov obljublja izboljšanje produkcijskega okolja, vendar to doslej še ni bilo kvantitativno raziskano. Nedavni napredek v strojnem vidu, ki temelji na globokem učenju, prav tako še ni bil v celoti izkoriščen. Namen te študije je predstaviti arhitekturne in izvedbene podrobnosti najsodobnejšega sistema za pomoč pri montaži, ki temelji na modelu zaznavanja objektov. Predlagana arhitektura naj bi predstavljala merilo za sodobne podporne sisteme. Možnost preprečevanja napak je ugotovljena v študiji primera, v kateri testni subjekti ročno sestavijo zahtevno, proti eksploziji varno cevno svetilko. Rezultati kažejo 51 \% manj napak pri montaži v primerjavi s kontrolno skupino brez pomoči. Tri od štirih obravnavanih vrst napak so se zmanjšale za najmanj $42 \%$. Sistem bo najverjetneje preprečil zlasti napake zaradi izpustitve sestavnih delov. Zmanjšanje pogostosti napak je opaziti v celotnem obdobju 30 zaporednih sestavljanj izdelka, če primerjamo montažo s pomočjo sistema in brez njega. Poleg tega se je izkazalo, da so zabeleženi podatki o montaži dragoceni tudi zaradi sledljivosti in izboljšanja proizvodnje.

\section{PODATKI O ČLANKU}

Ključne besede:

Globoko učenje;

Strojno učenje;

Industrija 4.0;

Pametna proizvodnja;

Ročna montaža;

Podporni sistem;

Preprečevanje napak;

Zaznavanje predmetov

*Kontaktna oseba:

alexander.riedel@eah-jena.de

(Riedel, A.)

Zgodovina članka:

Prejet 14. oktobra 2021

Popravljen 3. decembra 2021

Sprejet 4. decembra 2021

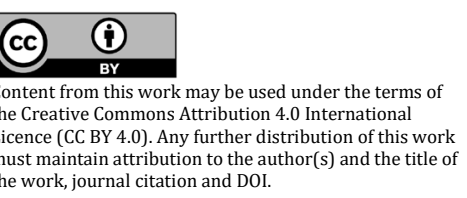

